\title{
Spectroscopy of the Pulsar-like White Dwarf AE Aquarii from Chandra and Swift XRT Data
}

\author{
Bosco Oruru *it \\ University of the Free State \\ E-mail: OruruBdufs.ac.za

\section{P. J. Meintjes} \\ University of the Free State \\ E-mail: Meint jPJ@ufs.ac.za
}

\begin{abstract}
The multi-wavelength properties of the highly transient novalike variable AE Aqr makes it an ideal laboratory for the study of accretion related astrophysical fluid dynamics. It consists of a fast rotating highly magnetic white dwarf (WD) orbiting, and accreting mass, from a low-mass main sequence companion. The WD is spinning down at a rate $\dot{p} \sim 5.64 \times 10^{-14} \mathrm{~s} \mathrm{~s}^{-1}$, corresponding to a spin-down luminosity of $L_{\mathrm{sd}} \sim 6 \times 10^{33} \mathrm{erg} \mathrm{s}^{-1}$. The system is in a propeller state, and most of its emission properties are associated with the propeller process. It has been detected in almost all wavelengths, with the optical emission dominated by emission from the companion. We have analysed its X-ray spectra using contemporanous Chandra and Swift X-ray data. The results of this study show that the X-ray emission has both thermal and non-thermal characteristics. The associated energy $(k T)$ range is $\sim 0.2-3.8 \mathrm{keV}$ and power-law index for the non-thermal part is $p \sim 2.2$. In this paper, results related to the Chandra and Swift X-ray spectroscopy, based on the constraints of the thermal and non-thermal emission mechanisms, will be presented.
\end{abstract}

Keywords: Binary stars: individual (AE Aquarii) - Stars: White dwarf - Accretion: Magnetic

Fast $X$-ray timing and spectroscopy at extreme count rates

February 7-11, 2011

Champéry, Switzerland

\footnotetext{
${ }^{*}$ Speaker.

†The content of this paper is part of the work intended for the author's PhD thesis under the supervision of the co-author, Prof P. J. Meintjes.
} 


\section{Introduction}

AE Aquarii (hereafter AE Aqr) is a low mass non-eclipsing close binary system consisting of a magnetic white dwarf, accreting matter from a late-type main sequence companion (e.g. Welsh et al. 1995; Ikhsanov 1997; Choi et al. 1999). The orbital period is $9.88 \mathrm{~h}$ (e.g. Welsh et al. 1993). It has traditionally been classified as a novalike variable (e.g. Joy 1954; Crawford \& Craft 1956; de Jager 1991). Based on this classification, Patterson (1979) adopted the oblique rotator model and explained the system in terms of a magnetized white dwarf $\left(P_{\text {spin }} \sim 33 \mathrm{~s}\right)$ accreting matter from a well developed accretion disc, which placed AE Aqr in the intermediate polar subclass of the cataclysmic variables (e.g. Warner 1983; Ikhsanov 1997). However, the observed spectral profile of the $\mathrm{H}_{\alpha}$ emission line is single-peaked and the spectral widths of the Balmer emission lines are highly variable (e.g. Itoh et al. 2006, and references therein), seriously questioning the presence of an accretion disc.

AE Aqr has been detected in almost all wavelengths (e.g. de Jager 1991); in radio (e.g. Bookbinder \& Lamb 1987), in optical (e.g. Zinner 1938; Patterson 1979), in X-rays (e.g. Patterson et al. 1980; Clayton \& Osborne 1995) and possibly in $\mathrm{TeV} \gamma$-rays (e.g. Meintjes et al. 1992, 1994). In radio and $\mathrm{TeV} \gamma$-rays, the emission is reportedly of non-thermal nature (e.g. Ikhsanov 1997), and the rest of the emission is predominantly thermal. Coherent oscillations at $33 \mathrm{~s}$ were first detected in the optical light (e.g. Patterson 1979), and later in other wavelengths (e.g. Patterson et al. 1980; de Jager et al. 1994; Eracleous et al. 1994; Meintjes et al. 1994).

Recent studies (e.g. Wynn et al. 1997; Meintjes \& de Jager 2000) have shown that the properties of the emission lines can be explained satisfactorily within the magnetic propeller model. These authors have shown that the fast rotating white dwarf will most probably act as a magnetospheric propeller, ejecting the bulk of

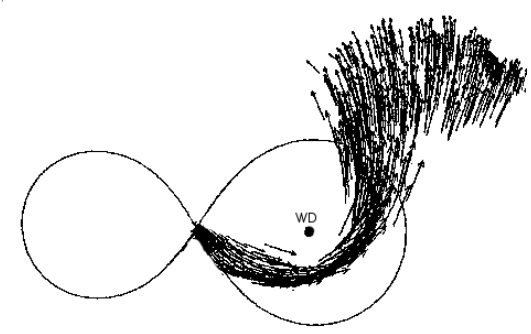

the mass transfer flow from the binary system (see figure on the right; adapted from Wynn et al. 1997). For an effective propeller, no accretion disc is formed, a characteristic that makes AE Aquarii quite unique. The propeller model has been widely considered to account for the spin-down of the WD (de Jager et al. 1994; Mauche 2006) at $\dot{P} \sim 5.64 \times 10^{-14} \mathrm{~s} \mathrm{~s}^{-1}$. On the other hand, Meintjes \& de Jager (2000) have suggested that a fraction of the spin-down energy is channeled into particle acceleration and non-thermal emission.

This paper is organized as follows. In §2, observations of AE Aqr with Chandra and Swift are presented. A brief description of data reduction procedures, with respect to the spectra, are presented. The spectral properties are presented in $\S 3$, with a discussion of possible X-ray emission models presented in $\S 4$.

\section{Observations and Data Reduction}

AE Aqr was observed by Chandra (ObsID 5431) on August 30, 2005 at 06:37 UT for $\sim 80 \mathrm{ks}$ (e.g. Mauche 2006), using the ACIS-S detector and HETG. Standard processing was done at the 
Chandra X-ray Center (CXC), and data was acquired through HEASARC on-line service, provided by the NASA's Goddard Space Flight Center. To generate spectra, CIAO software was used, where the level 2 event file was used as input to create a spectrum file (pha2.fits) that was then split into single spectrum files with respect to the diffraction order and the grating arm. Then, for each of the single spectra, a response matrix file (RMF) was created and used alongside other data files to create the corresponding ancilliary response file (ARF).

Swift observed AE Aqr (target ID = 30295) between August 30 and September 2, 2005 for $\sim 10.5 \mathrm{ks}$, as a pre-planned target (PPT; Evans et al. 2009). The data were processed at the UK Swift Science Data Centre (UKSSDC) in April 2007. The X-Ray Telescope (XRT; Burrows et al. 2005) is one of the three science instruments on-board Swift. It is a sensitive, flexible and autonomous X-ray imaging spectrometer designed to measure fluxes, spectra and lightcurves over a wide dynamic range covering more than 7 orders of magnitude in flux. It is designed for an autonomous operation, switching between four different readout modes, depending on the source intensity (Hill et al. 2004; Evans et al. 2007). The results presented in this paper are based on data collected when the XRT was operating in the Photon Counting (PC) mode, in which full imaging and spectroscopic resolutions are retained, but timing resolution is limited to $2.5 \mathrm{~s}$ (Burrows et al. 2005). The data used to generate the spectra were obtained using the on-demand software through the HEASARC on-line service. These were acquired by mannually registering the information of AE Aqr. Detailed description is given in the original paper by Evans et al. (2009).

\section{Results}

\subsection{Chandra Spectra}

Figure 1 shows the background subtracted energy spectra of the HEG arm for the diffraction orders $m=-1$ (left) and $m=+1$ (right) respectively. Each of the spectra was generated using sherpa and fitted with a two-temperature vmekal (thermal emission) model, $k T \sim 0.65,3.37 \mathrm{keV}$ for $m=$ 1 and $k T \sim 0.67,3.32 \mathrm{keV}$ for $m=+1$ respectively. In Figure 2, the corresponding MEG spectra are shown. Each spectrum is fitted with a three-temperature vmekal model, $k T \sim 0.20,0.68,3.82 \mathrm{keV}$ for $m=-1$ and $k T \sim 0.40,0.82,3.83 \mathrm{keV}$ for $m=+1$ respectively. From the figures, a number of emission lines are seen superimposed on the continuum emission, and most of which are prominent in the soft X-ray regime.

The average fluxes of X-ray emission for the energy range 0.3-10 keV were found to be $\sim$ $8.09 \times 10^{-12} \mathrm{ergs} \mathrm{cm}^{-2} \mathrm{~s}^{-1}$ and $1.04 \times 10^{-11} \mathrm{ergs} \mathrm{cm}^{-2} \mathrm{~s}^{-1}$ for HEG and MEG respectively. For an estimated source distance of $100 \mathrm{pc}$ (e.g. Welsh et al. 1993), then it can be shown that the X-ray luminosities are $\sim 9.71 \times 10^{30} \mathrm{erg} \mathrm{s}^{-1}$ and $1.25 \times 10^{31} \mathrm{erg} \mathrm{s}^{-1}$ for HEG and MEG respectively.

\subsection{Swift-XRT Spectra}

Figure 3 (left) shows the spectrum of AE Aqr from Swift-XRT data, generated in XSPEC. Fitting was performed using the C-statistic, and the resulting parameters were obtained with 90 $\%$ confidence for which the fit was repeatedly done until the C-statistic had worsened by 2.706 compared with the best fitting value. The spectrum was fitted with a combination of an absorbed power-law ( $p \sim 2.19 \pm 0.16)$ and a two-temperature vmekal emission model $\left(k T_{1} \sim 0.44 \pm 0.10 \mathrm{keV}\right.$ 

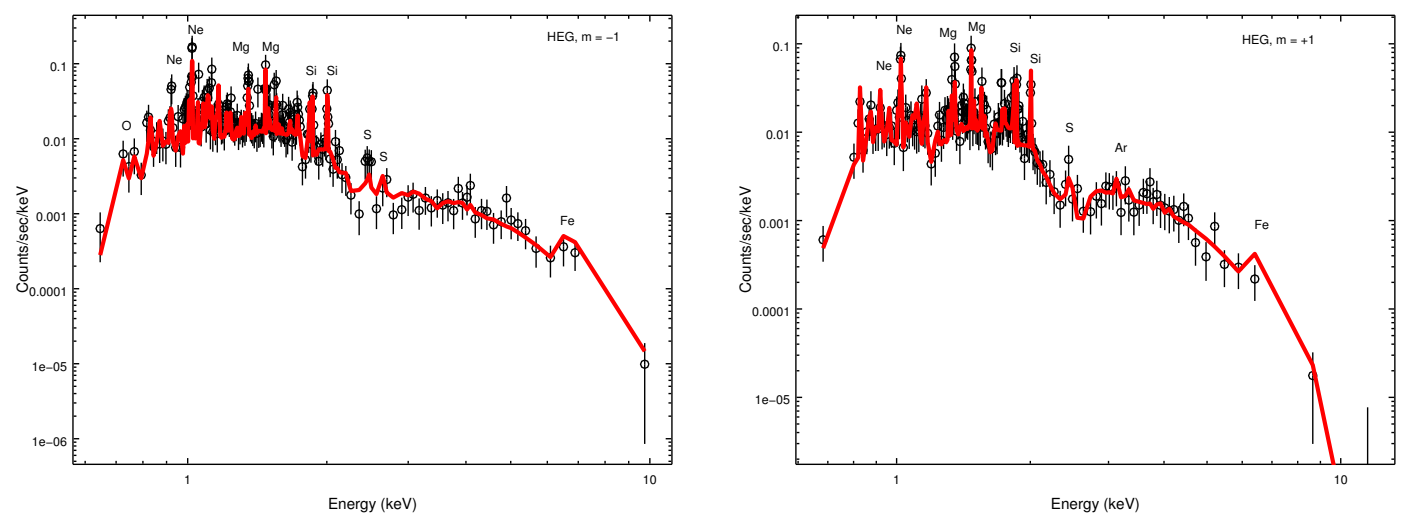

Figure 1: Left: HEG spectrum for the diffraction order $m=-1$. Right: HEG spectrum for the diffraction order $m=+1$.
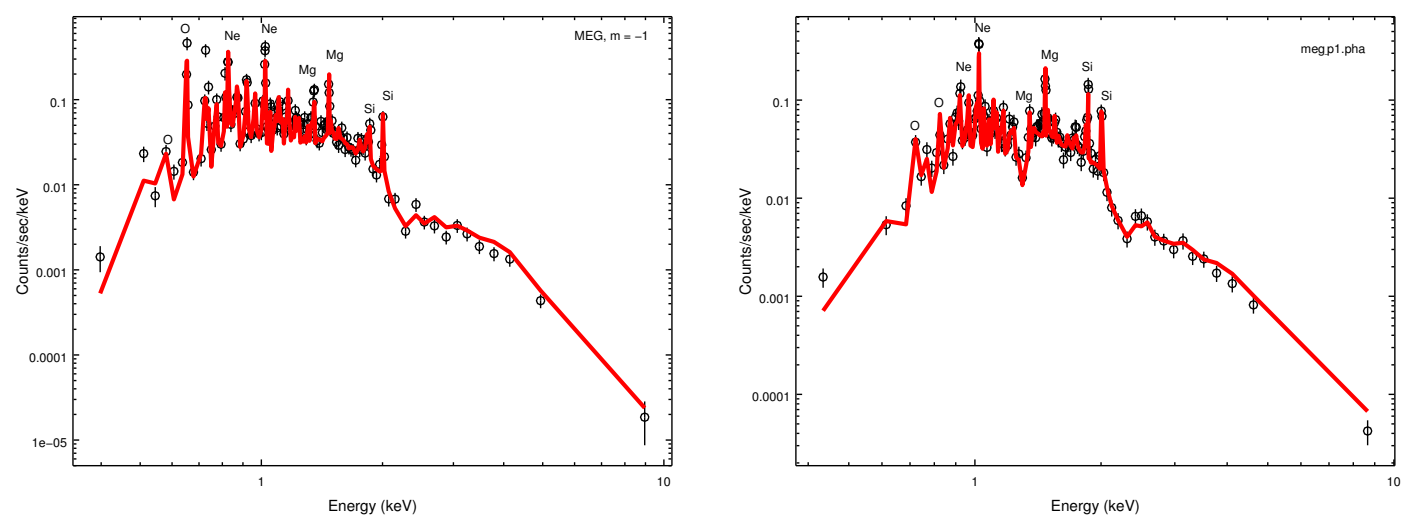

Figure 2: Left: MEG spectrum for the diffraction order $m=-1$. Right: MEG spectrum for the diffraction order $m=+1$.

and $k T_{2} \sim 0.96 \pm 0.11 \mathrm{keV}$ respectively), suggesting that the $\mathrm{X}$-ray emission has both thermal and non-thermal characteristics. On the right is the unfolded models plotted with the data. The blue and red dotted lines are the vmekal models, showing emission lines prominent at lower X-ray energies $(\lesssim 2.5 \mathrm{keV}$ ). The power-law model (green dashed line) provides a satisfactory fit for data in the hard energy range $(\mathrm{kT}>2 \mathrm{keV})$. Plots of photon and energy fluxes (Figure 4) also show that the hard X-ray data can be fitted with a power-law $(p \sim 2.01)$.

The average flux obtained for the default Swift-XRT soft energy range $(0.3-1.5 \mathrm{keV})$ was $\sim$ $6.22 \times 10^{-12} \mathrm{erg} \mathrm{cm}^{-2} \mathrm{~s}^{-1}$, and the corresponding default hard X-ray (1.5-10 keV) flux was $4.68 \times$ $10^{-12} \mathrm{erg} \mathrm{cm}^{-2} \mathrm{~s}^{-1}$. Then, the luminosities in the soft and hard energy bands are $7.46 \times 10^{30} \mathrm{erg}$ $\mathrm{s}^{-1}$ and $5.62 \times 10^{30} \mathrm{erg} \mathrm{s}^{-1}$ respectively. Thus, the total X-ray luminosity is $\sim 1.31 \times 10^{31} \mathrm{erg} \mathrm{s}^{-1}$.

\section{Discussions}

The spectral analyses of both Chandra and Swift-XRT data show that the X-ray emission is predominantly soft and contains a number of emission lines. These characteristics are associated 

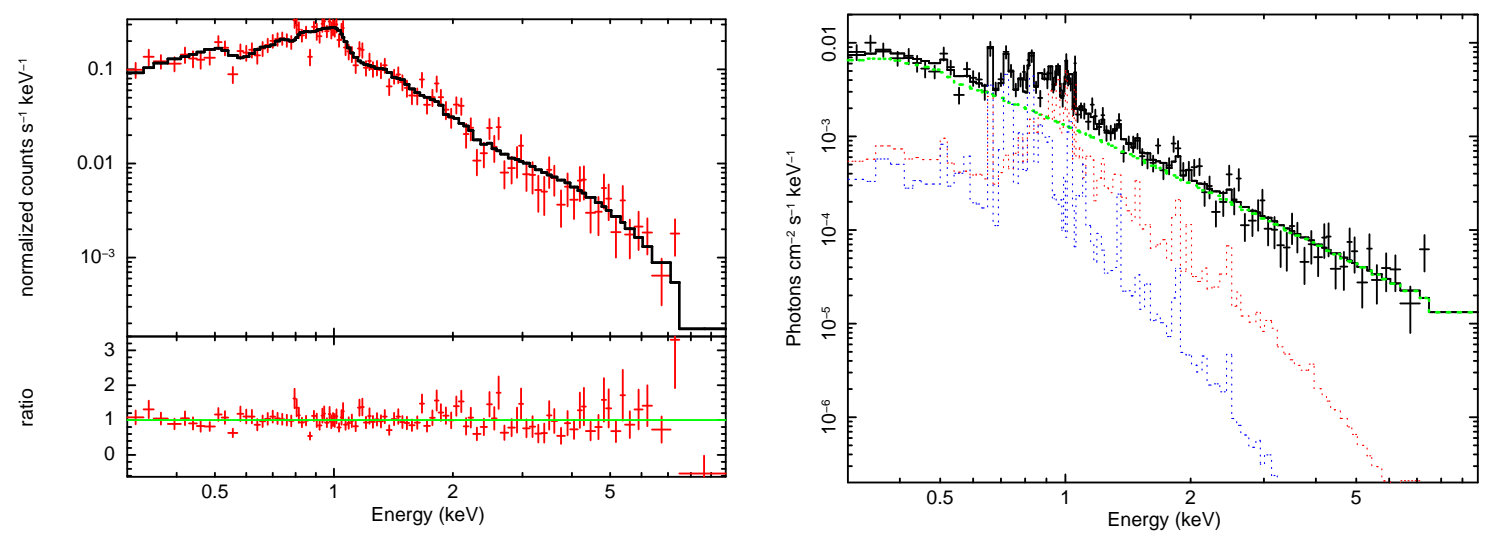

Figure 3: Left: Spectrum of AE Aquarii with the best fitting models. Right: Unfolded models plotted with data.
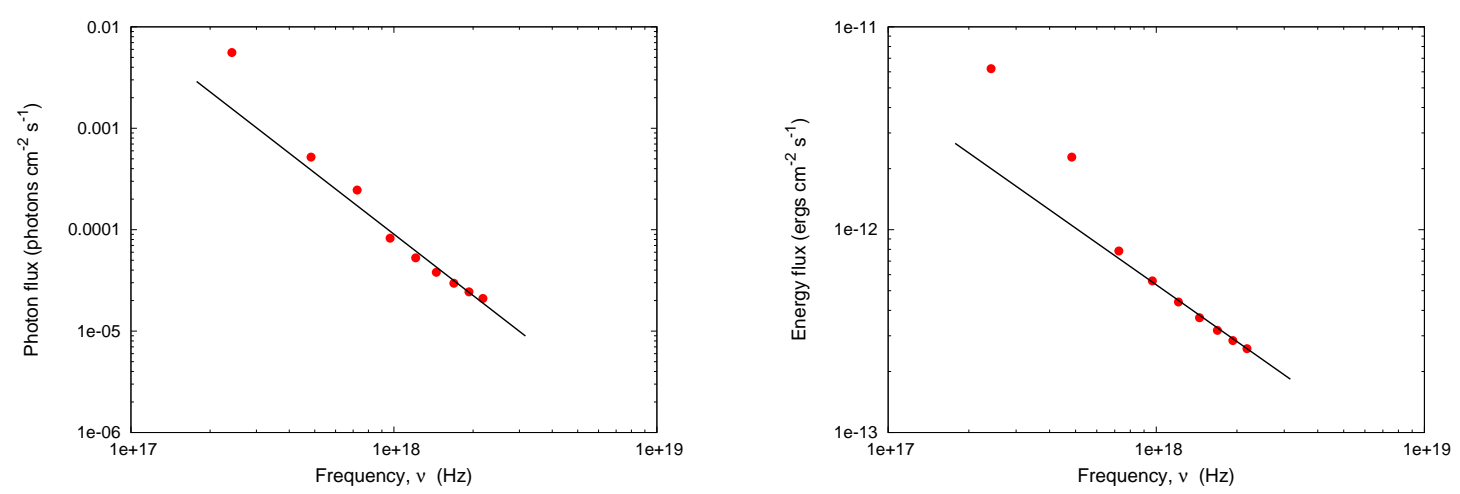

Figure 4: Photon flux (left) and energy flux (right), fitted with a power-law of index, $p \sim 2.01$.

with an optically thin emission region with a continuous temperature distribution. The vmekal emission model (with $T>10^{6} \mathrm{~K}$ ) means that a thermal emission process is one of the components of the X-ray emission of AE Aqr. Black body emission is unlikely because the observed luminosity $\left(\sim 10^{31} \mathrm{erg} \mathrm{s}^{-1}\right)$ is much less than $\sim 10^{34} \mathrm{erg} \mathrm{s}^{-1}$, which is the expected value for accretion onto the surface of the white dwarf. For AE Aqr, the very effective propeller process greatly hinders the accretion onto the surface, and most likely then, bremsstrahlung (from heated mass outflow) could be the thermal emission process responsible for most of the X-rays (e.g. Oruru \& Meintjes, in preparation). With a temperature, $T>10^{6} \mathrm{~K}$, it would require that the X-ray emitting region is well above the polar cap region (e.g. Patterson et al. 1980).

The power-law model, used alongside the vmekal model in fitting the Swift-XRT data, suggests a non-thermal emission, possibly arising from synchrotron radiation of accelerated particles (e.g. Terada et al. 2008). This is suggested for the hard X-ray emission which is modelled to have a different emission region from the soft X-ray emission, although both components are pulsed (e.g. Oruru \& Meintjes, in preparation). It is proposed that the hard X-ray emission should be from a region in the vicinity of the light cylinder radius (e.g. Oruru \& Meintjes, in preparation).

The spectral characteristics of AE Aqr discussed above make it similar to accretion-driven X-ray pulsars, but with properties of the white dwarf (e.g. spin period and surface magnetic field) much different compared with those of neutron stars. Hence, the hard X-ray emission in AE Aqr is 
not strong enough to be detected by most X-ray observatories.

Acknowledgements: This work made use of data supplied by the Chandra X-ray Center, and the UK Swift Science Data Centre at the University of Leicester. The research has been funded by the African Square Kilometre Array Project, and the National Research Foundation of South Africa.

\section{References}

[1] Bookbinder J. A., Lamb D. Q., 1987, ApJ, 323, 131

[2] Burrows D. N., Hill J. E., Nousek J. A. et al., 2005, Space Sci. Rev., 120, 165

[3] Choi C-S., Dotani T., Agrawal P. C., 1999, ApJ, 525, 399

[4] Clayton K. L., Osborne J. P., 1995, ASPC, 85, 379C

[5] Crawford J. A., Craft R. P., 1956, ApJ, 123, 44

[6] de Jager O. C., 1991a, ApJ, 378, 286

[7] de Jager O. C., 1991b, ICRC, 2, 463

[8] de Jager O. C., Meintjes P. J., O’Donoghue D., Robinson E. L., 1994, MNRAS, 267, 577

[9] Eracleous M., Horne K., Robinson E.L., Zhang E., Marsh T.R. \& Wood J.H., 1994, ApJ, 433, 313

[10] Evans P. A., Beardmore A. P., Page K. L., Osborne J. P. et al., 2007, A\&A, 469, 379

[11] Evans P. A., Beardmore A. P., Page K. L., Osborne J. P., et al., 2009, MNRAS, 397, 1177

[12] Hill J. E., Burrows D. N., Nousek J. A., et al. 2004, SPIE, 5165, 217

[13] Ikhsanov N. K., 1997, A\&A, 325, 1045

[14] Itoh K., Okada S., Ishida M., Kunieda H., 2006, ApJ, 639, 397

[15] Mauche C. W., 2006, MNRAS, 369, 1983

[16] Meintjes P. J., de Jager O. C., 2000, MNRAS, 311, 611

[17] Meintjes P. J., de Jager O. C., Raubenheimer B. C., Nel H. I., et al., 1994, ApJ, 434, 292

[18] Meintjes P.J., Raubenheimer B.C., de Jager O.C., Brink C., et al., 1992, ApJ, 401, 325

[19] Oruru B., Meintjes P. J., 2011, in preparation, MNRAS

[20] Patterson J., 1979, ApJ, 234, 978

[21] Patterson J., Branch D., Chincarini G., Robinson E. L., 1980, ApJ, 240, L133

[22] Terada Y., Ishida M., Mukai K., Dotani T., et al., 2008, Adv. Space Research, 41, 512

[23] Warner B., 1983, in Livio M., and Shaviv G. (eds.), Cataclysmic Variables and Related Objects, Dordrecht: Reidel, p. 155

[24] Welsh W. F., Horne K., Gomer R., 1993, ApJ, 410, L39

[25] Welsh W. F., Horne K., Oke J.B., 1993, ApJ, 406, 229

[26] Welsh W. F., Horne K., Gomer R., 1995, MNRAS, 275, 649

[27] Wynn G. A., King R. A., Horne K., 1997, MNRAS, 286, 436

[28] Zinner E., 1938, Astron.Nach., 265, 345 